$11 ; 15$

\title{
Матричное радиовидение на основе гетеродинного приема с применением радиолокации непрерывным излучением
}

\author{
(C) С.А. Королев ${ }^{1}$, А.В. Горюнов ${ }^{1}$, В.В. Паршин ${ }^{2}$ \\ ${ }^{1}$ Институт фризики микроструктур РАН, Афонино, Нижегородская обл., Россия \\ ${ }^{2}$ Институт прикладной фризики РАН, Нижний Новгород, Россия \\ E-mail: pesh@ipm.sci-nnov.ru
}

Поступило в Редакцию 13 августа 2021 г.

В окончательной редакции 27 сентября 2021 г.

Принято к публикации 27 сентября 2021 г.

\begin{abstract}
Предложен новый подход к созданию систем радиовидения миллиметрового диапазона. Данный подход основан на использовании приемной матрицы, состоящей из плотноупакованного (размер пикселя $4 \mathrm{~mm}$ ) массива планарных смесителей, расположенных в фокальной плоскости квазиоптического объектива, с применением метода радиолокации непрерывным излучением с частотной модуляцией. Продемонстрировано, что реализация гетеродинного типа приема позволяет увеличить дальнодействие матричной системы радиовидения до $\sim 100 \mathrm{~m}$ с сохранением угловой разрешающей способности на прежнем уровне.
\end{abstract}

Ключевые слова: миллиметровые волны, радиовидение, матричный приемник, радиолокация непрерывным излучением с частотной модуляцией.

DOI: 10.21883/PJTF.2022.01.51880.18992

Системы радиовидения миллиметрового диапазона сочетают в себе высокое разрешение с низким уровнем поглощения в атмосфере и многих других средах и материалах [1,2]. По этой причине диапазон миллиметровых волн во многих случаях представляет собой оптимальный радиодиапазон для задач радиовидения.

Наиболее простую конструкцию имеют приемные системы, построенные на принципе прямого детектирования, когда измеряется только амплитуда радиосигнала $[3,4]$. Выходное напряжение приемника при этом пропорционально мощности принимаемого сигнала, которая имеет достаточно резкую зависимость от расстояния $R$, пропорциональную $1 / R^{4}$. Быстрый спад уровня выходного сигнала с увеличением расстояния определяет дальность действия систем детекторного типа на уровне нескольких метров, что оказывается недостаточным для ряда задач.

Более чувствительными являются приемники гетеродинного типа, выходное напряжение которых определяется амплитудой принимаемого сигнала, смешиваемого с сигналом гетеродина. Такие приемники нашли широкое применение в радиолокации и радиосвязи, однако при построении матричных систем радиовидения использование гетеродинного типа приема приводит к серьезным трудностям, связанным с созданием плотного массива приемных элементов и распределения мощности гетеродина между большим числом элементов.

В настоящей работе предложена и экспериментально исследована возможная реализация матричной системы радиовидения гетеродинного типа, определены ее достижимые характеристики.

В качестве базового подхода к созданию системы радиовидения гетеродинного типа был выбран метод радиолокации непрерывным излучением с линейной частотной модуляцией [5-7]. Данный подход основан на том, что в качестве сигнала гетеродина используется часть мощности (реплика) передаваемого излучения. Таким образом, принимаемый и опорный сигналы когерентны, что исключает влияние фазового шума генератора на качество приема. За счет частотной модуляции принимаемый и опорный сигналы различаются по частоте, поэтому на выходе смесительного элемента требуется усиливать не постоянный сигнал, а сигнал разностной частоты, что во многих случаях оказывается более удобным. При этом по значению разностной частоты может быть определено расстояние до отражателя, что открывает возможности для получения трехмерного изображения сцены.

На рис. 1 представлено схематическое изображение исследуемой системы. В качестве источника миллиметрового излучения используется генератор на основе лампы обратной волны $\mathrm{W}$-диапазона. Вблизи рабочей частоты $94 \mathrm{GHz}$ мощность генератора составляет $10 \mathrm{~mW}$. Использовалась пилообразная форма частотной модуляции с размахом $B=576 \mathrm{MHz}$. С помощью направленного ответвителя мощность генератора практически поровну разделяется на две части. Одна из этих частей поступает на коническую рупорную антенну для засветки сцены, а вторая часть используется в качестве сигнала гетеродина приемника. Изображение сцены в плоскости расположения матричного приемника формируется квазиоптическим объективом, представляющим собой асферическую линзу диаметром $10 \mathrm{~cm}$ [8]. Фокусное расстояние линзы составляет $100 \mathrm{~mm}$. Фокальное пятно линзы имеет размер около $4 \mathrm{~mm}$, что соответствует размеру пикселя приемной матрицы. Облучение 


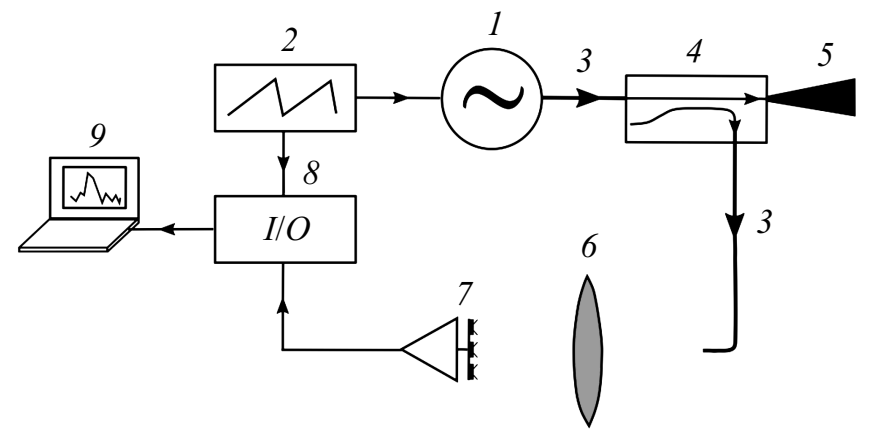

Рис. 1. Схематическое изображение системы радиовидения гетеродинного типа. 1 - генератор W-диапазона на основе лампы обратной волны, 2 - генератор сигналов специальной формы, 3 - металлические волноводы, 4 - направленный ответвитель, 5 - коническая рупорная антенна, $6-$ диэлектрическая линза, 7 - трехэлементный матричный приемник, 8 - устройство ввода/вывода, 9 - ноутбук.

матрицы сигналом гетеродина осуществляется непосредственно из открытого конца волновода, помещенного в фокусе диэлектрической линзы со стороны сцены. Таким образом реализуется равномерная по амплитуде и фазе засветка матрицы опорным излучением.

Для исследования базовых характеристик разрабатываемой системы использовался матричный приемник [9], состоящий из трех планарных смесительных элементов, расположенных в линию (рис. 2). Конструкция каждого приемного элемента в целом повторяет конструкцию одноэлементного приемника, представленного в [10]. Основой приемного элемента служит планарная структура, состоящая из низкобарьерного диода Шоттки, вмонтированного в модифицированную щелевую антенну. Размер пикселя матрицы составляет $4 \mathrm{~mm}$ в обоих направлениях. Исследованию характеристик системы предшествовала ее калибровка, в ходе которой программным путем устранялась неоднородность чувствительности приемных элементов, а также неоднородность засветки приемных элементов опорным излучением.

Предлагаемый метод радиовидения позволяет получать трехмерные изображения сцены: двумерное изображение формируется квазиоптическим объективом, а информация о дальности до объектов извлекается за счет анализа фазы сигнала разностной частоты. Таким образом, необходимо рассмотреть две величины, характеризующие разрешающую способность системы: угловую разрешающую способность и радиальную разрешающую способность.

Для исследования угловой разрешающей способности использовались два идентичных отражателя, расположенных на одинаковом расстоянии от объектива системы $(R=374 \mathrm{~cm})$. Отражатель представляет собой выпуклую асферическую металлизированную поверхность диаметром $10 \mathrm{~cm}$, базирующуюся на диэлектрических стойках. В начальном положении два отражателя находились максимально близко друг от друга; при этом полезный сигнал присутствовал только в центральном приемном элементе. При изменении углового расстояния между отражателями (с шагом $0.6^{\circ}$ ) сигнал из центрального приемного элемента переходит в боковые приемные элементы, а затем выходит за пределы угла обзора матрицы. Два отражателя становятся отличимыми при угле между ними $3.1^{\circ}$. В данном положении амплитуда боковых приемных элементов примерно в 2-3 раза больше, чем амплитуда центрального приемного элемента. Таким образом, установленная угловая разрешающая способность системы составляет не хуже $3^{\circ}$.

Радиальная разрешающая способность была изучена с использованием похожего расположения системы радиовидения и отражателей. Отличие состояло в том, что отражатели располагались на постоянном (минимально возможном) угловом расстоянии друг от друга, а радиальное расстояние между ними менялось (один из отражателей перемещался по направлению к системе радиовидения). В эксперименте был задействован только центральный приемный элемент. Когда отражатели находятся близко друг от друга, в спектре выходного сигнала приемного элемента имеется только одна линия на частоте, соответствующей среднему расстоянию до отражателей. Начиная с некоторого расстояния между отражателями линия спектра разделяется на две, каждая из которых соответствует своему отражателю. Было показано, что отражатели становятся различимыми при радиальном расстоянии между ними, равном $z=30 \mathrm{~cm}$. Данное значение находится в хорошем согласии со значением $\delta R=26 \mathrm{~cm}$, полученным из общеизвестного выражения для радиальной разрешающей способности радиолокатора непрерывного излучения с линейной частотной модуляцией $\delta R=c /(2 B)[5]$, где $c$ - скорость света в вакууме.

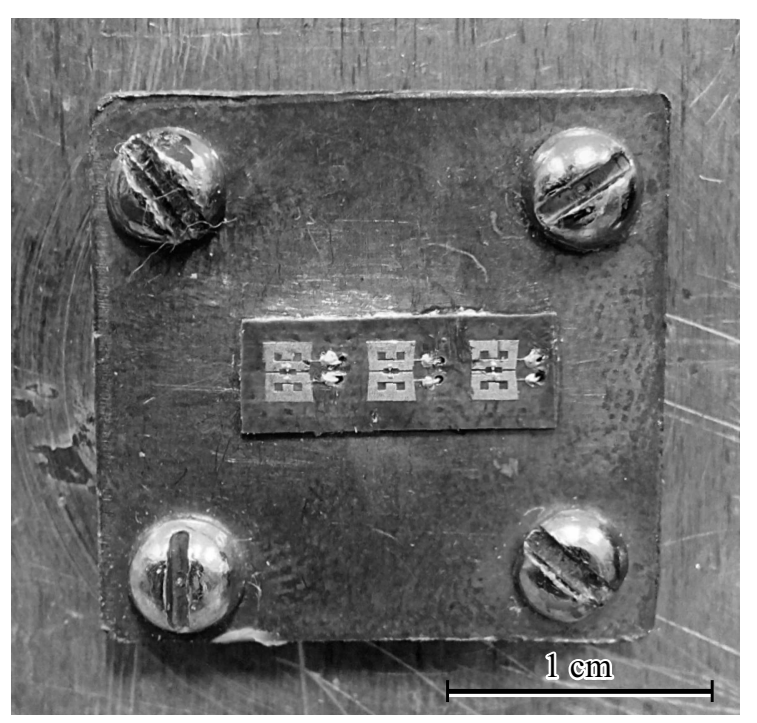

Рис. 2. Фотография высокочастотной части трехэлементного матричного приемника. 


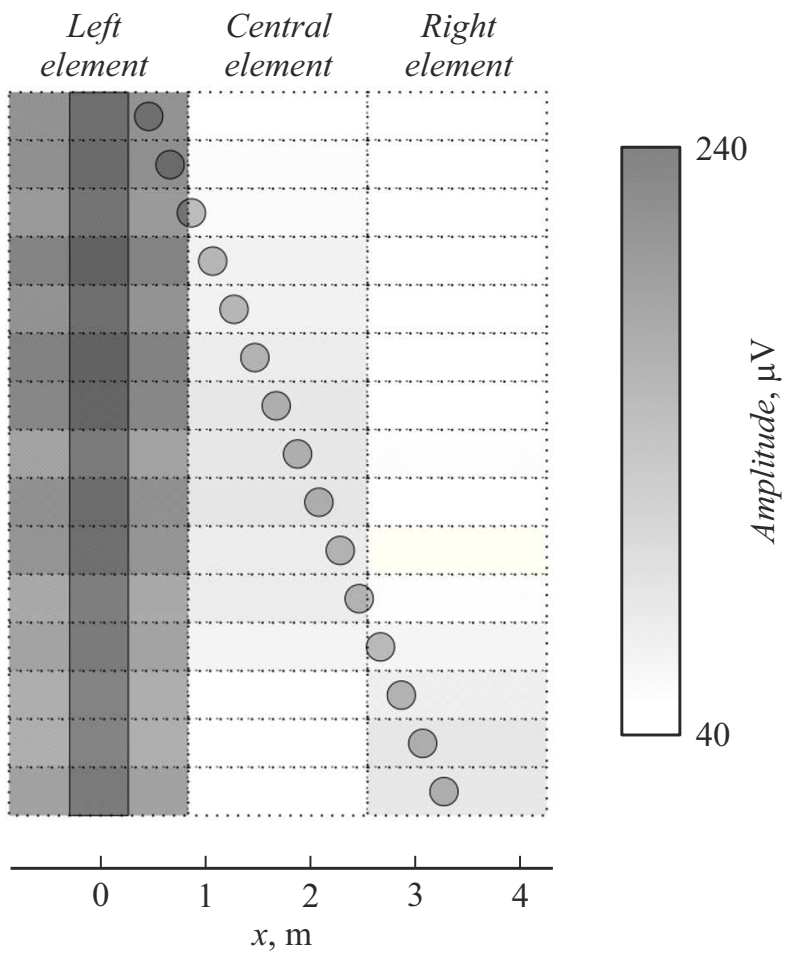

Рис. 3. Амплитуда сигнала разностной частоты в приемных элементах матричного приемника в зависимости от положения сферического отражателя $x$. Затемненный прямоугольник столб, затемненные кружки - отражатель в различных положениях. Пунктирные линии условно разделяют область пространства на регионы, отвечающие каждому из трех пикселей матрицы.

Дальнодействие системы радиовидения исследовалось в длинном коридоре. Система располагалась в одном конце коридора, отражатель с помощью передвижной треноги перемещался от места расположения системы к другому концу коридора. Экспериментальные данные получены до расстояния $40 \mathrm{~m}$, при котором уровень полезного сигнала сравнялся с уровнем сигнала от нежелательных переотражений, вызванных в основном отражениями от стен, потолка и пола. При более удачных условиях эксперимента может быть достигнуто дальнодействие до $100 \mathrm{~m}$, определяемое уровнем шума приемника.

В описанных экспериментах угловое разрешение и дальнодействие были исследованы независимо: угловое разрешение измерялось на коротком расстоянии, а дальнодействие исследовалось в режиме одноэлементного приема. Для проверки сочетания достигнутых характеристик был поставлен демонстрационный эксперимент, в котором одновременно тестируются оба параметра: эксперимент посвящен различению малого объекта на фоне крупного на расстоянии несколько десятков метров.

Конфигурация эксперимента следующая. Система радиовидения располагается напротив открытого окна на втором этаже здания. Единственный отражающий объект, который попадает в поле зрения системы радиовидения, - столб диаметром $0.6 \mathrm{~m}$. Расстояние от объектива системы до столба составляет $44 \mathrm{~m}$. Приемная матрица располагается в фокальной плоскости линзы таким образом, чтобы максимальная амплитуда сигнала разностной частоты достигалась на левом приемном элементе. В других приемных элементах полезный сигнал при этом отсутствует. Далее на столб с помощью тонкого деревянного бруска был прикреплен отражатель в виде шарового сегмента с радиусом закругления $60 \mathrm{~cm}$ и диаметром основания $30 \mathrm{~cm}$. Начальное расстояние между центром столба и центром отражателя составляло $x=50 \mathrm{~cm}$ (первая строка на рис. 3). На каждом шаге измерений отражатель отодвигался от столба на $\Delta x=20 \mathrm{~cm}$ и записывались амплитудные спектры сигналов разностной частоты каждого приемного элемента (рис. 3). Конечное расстояние составило $x=330 \mathrm{~cm}$ (последняя строка на рис. 3). Результаты эксперимента показывают, что столб и перемещаемый отражатель становятся различимыми при $x>270 \mathrm{~cm}$. Для сопоставления с результатами эксперимента, проведенного внутри комнаты, данное значение нужно уменьшить на $30 \mathrm{~cm}$. Это объясняется тем, что краевые области столба также участвуют в отражении сигнала, поэтому реальное расстояние между объектами в данном случае представляет собой расстояние между их границами, а не центрами. Таким образом, минимальный угол, при котором столб и отражатель различимы, составляет $\theta=3.2^{\circ}$, что находится в хорошем согласии со значением, полученным в эксперименте на коротком расстоянии.

Таким образом, в работе предложен и экспериментально продемонстрирован новый подход к созданию систем радиовидения миллиметрового диапазона. За счет использования гетеродинного типа приема сигнала удается существенно повысить чувствительность матричного приемника и соответственно увеличить дальнодействие систем радиовидения более чем на порядок. Это позволяет рассчитывать на возможность создания систем миллиметрового радиовидения с рабочим расстоянием до нескольких сотен метров.

\section{Благодарности}

В работе использовано оборудование ЦКП ИФМ РАН „Физика и технология микро- и наноструктур“.

\section{Финансирование работы}

Исследование выполнено за счет гранта Российского научного фонда (проект № 20-79-00128).

\section{Конфликт интересов}

Авторы заявляют, что у них нет конфликта интересов. 


\section{Список литературы}

[1] L. Yujiri, M. Shoucri, P. Moffa, IEEE Microwave Mag., 4 (3), 39 (2003). DOI: 10.1109/MMW.2003.1237476

[2] P.F. Goldsmith, C.-T. Hsieh, G.R. Huguenin, J. Kapitzky, E.L. Moore, IEEE Trans. Microw. Theory Techn., 41 (10), 1664 (1993). DOI: $10.1109 / 22.247910$

[3] В.И. Шашкин, Ю.И. Белов, П.В. Волков, А.В. Горюнов, В.Р. Закамов, И.А. Илларионов, Письма в ЖТФ, 39 (12), 44 (2013).

[4] J.J. Lynch, H.P. Moyer, J.H. Schaffner, Ya. Royter, M. Sokolich, B. Hughes, Y.J. Yoon, J.N. Schulman, IEEE Trans. Microw. Theory Techn., 56 (7), 1592 (2008).

DOI: 10.1109/TMTT.2008.924361

[5] J.-M. Muñz-Ferreras, Z. Peng, R. Gómez-Garcia, G. Wang, C. Gu, C. Li, IEEE Microwave Mag., 16 (4), 40 (2015). DOI: 10.1109/MMM.2015.2393995

[6] D. Bleh, M. Rösch, M. Kuri, A. Dyck, A. Tessmann, A. Leuther, S. Wagner, B. Weismann-Thaden, H.-P. Stulz, M. Zink, M. Rießle, R. Sommer, J. Wilcke, M. Schlechtweg, B. Yang, O. Ambacher, IEEE Trans. Microw. Theory Techn., 65 (9), 3474 (2017). DOI: 10.1109/TMTT.2017.2661742

[7] Д.Я. Суханов, В.П. Якубов, ЖТФ, 80 (4), 115 (2010).

[8] П.В. Волков, Ю.И. Белов, А.В. Горюнов, И.А. Илларионов, А.Г. Серкин, В.И. Шашкин, ЖТФ, 84 (4), 120 (2014).

[9] S.A. Korolyov, A.P. Shikov, V.V. Parshin, in 2020 7th AllRussian Microwave Conf. (RMC) (IEEE, 2020), p. 15. DOI: $10.1109 /$ RMC50626.2020.9312250

[10] S.A. Korolyov, A.P. Shikov, A.V. Goryunov, V.I. Shashkin, IEEE Sensors Lett., 4 (5), 3500404 (2020).

DOI: $10.1109 /$ LSENS.2020.2986370 